\title{
Differential effects of antiepileptic drugs on human bone cells
}

\author{
Sara Rocha ${ }^{1,2}$ Ricardo Ferraz ${ }^{2,3}$ Cristina Prudêncio ${ }^{2,4}$ Maria Helena Fernandes ${ }^{1,3}$ João \\ Costa-Rodrigues ${ }^{1,5,6,7}$
}

${ }^{1}$ Laboratory for Bone Metabolism and Regeneration, Faculty of Dental Medicine, $U$. Porto, Portugal

${ }^{2}$ Ciências Químicas e das Biomoléculas (CQB) e Centro de Investigação em Saúde e Ambiente (CISA), Escola Superior de Saúde do Instituto Politécnico do Porto, Portugal

${ }^{3}$ REQUIMTE/LAQV, U. Porto, Portugal

${ }^{4}$ i3S, Instituto de Inovação e Investigação em Saúde, Universidade do Porto, Portugal

${ }^{5}$ ESS - Escola Superior de Saúde, P. Porto, Portugal

\author{
${ }^{6}$ Instituto Politécnico de Viana do Castelo, \\ Escola Superior de Saúde, Portugal \\ ${ }^{7}$ i3S, Instituto de Inovação e Investigação em \\ Saúde, Universidade do Porto, Portugal \\ Correspondence \\ João Costa-Rodrigues, Faculdade de Medicina \\ Dentária, Universidade do Porto, Rua Dr. \\ Manuel Pereira da Silva, 4200-393 Porto. \\ Email: jrodrigues@fmd.up.pt
}

\author{
Funding information \\ FEDER, Grant/Award Number: POCI/01/ \\ 0145/FEDER/007265; Fundação para a \\ Ciência e Tecnologia and Ministério da \\ Educação e Ciência, Grant/Award Number: \\ PT2020 UID/QUI/50006/2013; Comissão de \\ Coordenação e Desenvolvimento Regional do \\ Norte, Grant/Award Number: \\ Norte-01-0145-FEDER-000024
}

\section{Abstract}

Antiepileptic drugs (AED) have been associated to in vivo deleterious consequences in bone tissue. The present work aimed to characterize the cellular and molecular effects of five different AED on human osteoclastogenesis and osteblastogenesis. It was observed that the different drugs had the ability to differentially modulate both processes, in a way dependent on the identity and dose of the AED. Shortly, valproic acid stimulated either osteoclastogenesis and osteoblastogenesis, whereas carbamazepine, gabapentin, and lamotrigine revealed an opposite behavior; topiramate elicited a decrease of osteoclast development and an increase in osteoblast differentiation. This is the first report describing the direct effects of different AED on human primary bone cells, which is a very important issue, because these drugs are usually consumed in long-term therapeutics, with acknowledged in vivo effects in bone tissue.

KEYWORDS

antiepileptic drugs, osteoblastogenesis, osteoclastogenesis

\section{1 | INTRODUCTION}

Epilepsy is a common neurological disorder, affecting about 50 million people (Fan et al., 2016). Antiepileptic drugs (AED) are a widely used class of drugs, indicated for the treatment of epilepsy, convulsions, seizures, pain, neuropathy, and psychiatric disorders (Fan et al., 2016; Hant \& Bolster, 2016; Pitetzis, Spilioti, Yovos, \& Yavropoulou, 2017). Their intake is associated with a variety of side effects, including dizziness, drowsiness, mental slowing, skin rashes, hepatotoxicity, pancreatic insufficiency, behavioral disorders, increased risk of metabolic syndrome, and nephrolithiasis (Fan et al., 2016; Lin et al., 2016). In addition, since the late $60 \mathrm{~s}$ there is growing evidence pointing to a disruption of bone metabolism and a decreased bone mineral density, particularly in children and adolescents (Hant \& Bolster, 2016; Lin et al., 2016; Pitetzis et al., 2017; Rauchenzauner et al., 2010), supported by an increased risk of fracture, rickets, osteomalacia, osteoporosis, abnormal dentition, and decreased growth (Carbone et al., 2010; Lazzari et al., 2013; Shen, Chen, Zhang, Guo, \& Ding, 2014). In line with this, it was reported that valproic acid and lamotrigine and, especially, a combination of both, is associated with a decreased stature (Guo, Ronen, \& Atkinson, 2001), though the underlying mechanisms are not yet understood. The real effects of AED in bone tissue remain to be elucidated and are involved in some controversy, since some studies have failed to prove a consistent relationship, for example, between 
valproic acid and carbamazepine in bone development and growth (Rattya, Vainionpaa, Knip, Lanning, \& Isojarvi, 1999). AED mechanisms of action with a potential impact in bone tissue are diverse, including modulation of cytochrome P450 activity, inhibition of osteocalcin production/function, stimulation of vitamin D catabolism, and increased calcium excretion in the urine (Ecevit, Aydogan, Kavakli, \& Altinoz, 2004; Pitetzis et al., 2017). Globally, although there are many in vivo observations that point to a possible dysregulation of bone metabolism by AED, at the present, the data regarding the direct effects of those drugs on bone cells are still very scarce (Hatakeyama et al., 2011; Humphrey, Morris, \& Fuller, 2013; Schroeder \& Westendorf, 2005; Schroeder, Nair, Staggs, Lamblin, \& Westendorf, 2007).

Bone is a very dynamic tissue that is constantly being shaped molded, and remodeled by the coordinated action of two major cell types, the osteoclasts and the osteoblasts (Siddiqui \& Partridge, 2016). The former are specialized cells in bone resorption, which degrade bone extracellular matrix by two different mechanisms, namely, the secretion of $\mathrm{H}^{+}$that leads to acid erosion of bone mineral content, and the secretion of proteases, which digest extracellular matrix proteins, mainly collagen type 1 (Soysa \& Alles, 2016). On the other hand, osteoblasts synthesize new bone tissue, by lying down layers of collagen and other proteins, forming the so-called osteoid and, after that, depositing nanocrystals of hydroxyapatite (Valenti, Dalle Carbonare, \& Mottes, 2016). Imbalances between both activities can lead to abnormalities in bone composition, structure and, consequently, function.

The aim of the present work was to investigate whether different AED (valproic acid, carbamazepine, gabapentin, lamotrigine, and topiramate) have the ability to directly affect human osteoclastogenesis and osteoblastogenesis. For that, primary osteoclasts and osteoblasts were used, and cell cultures were characterized for different biochemical and molecular parameters, as well as for the involvement of several intracellular signaling pathways in cell response, in the presence of the tested AED.

\section{2 | MATERIALS AND METHODS}

\section{1 | Osteoclastic cell cultures}

Human peripheral blood mononuclear cells (PBMC) were used as osteoclastic precursors. They were isolated from the blood of healthy donors with 25-35 years old, after the informed consent, as described previously (Costa-Rodrigues, Carmo, Perpetuo, Monteiro, \& Fernandes, 2016b). Shortly, after dilution with phosphate-buffered saline (PBS) + $2 \mathrm{mM}$ EDTA (1:1), blood was applied on top of Ficoll-Paque ${ }^{\mathrm{TM}}$ PREMIUM (GE Healthcare Bio-Sciences, Chicago, Illinois, EUA) and centrifuged at $400 \mathrm{~g}$ for $30 \mathrm{~min}$. PBMC were collected, washed two times with PBS $+2 \mathrm{mM}$ EDTA and counted with a cytometer (Celltac MEK-5103, Nihon Kohden, Tokyo, Japan). Usually, for each $100 \mathrm{ml}$ of processed blood, about $70 \times 10^{6} \mathrm{PBMC}$ were obtained.

Cells were seeded at $1.5 \times 10^{6}$ cells $/ \mathrm{cm}^{2}$ in Minimum Essential Medium Eagle-alpha modification ( $\alpha$-MEM) supplemented with $30 \%$ autologous human serum, $100 \mathrm{IU} / \mathrm{ml}$ penicillin, $2.5 \mu \mathrm{g} / \mathrm{ml}$ streptomycin,
$2.5 \mu \mathrm{g} / \mathrm{ml}$ amphotericin B, and $2 \mathrm{mM}$ L-glutamine. Cell cultures were maintained for 21 days in a $5 \% \mathrm{CO}_{2}$ humidified atmosphere at $37^{\circ} \mathrm{C}$, and culture medium was replaced once a week.

Twenty-four hours after cell seeding, cells were treated with a wide range of the different tested AED $\left(10^{-8}-10^{-4} \mathrm{M}\right)$, which included the plasma levels normally achieved following the therapeutic usage of the drugs (Abou-Khalil, 2005; Haroutiunian, Ratz, Rabinovich, Adam, \& Hoffman, 2009; O'Dougherty, Wright, Cox, \& Walson, 1987; Popov, Maricic, Prosen, \& Voncina, 2013; Rose \& Kam, 2002). The tested AED were valproic acid, carbamazepine, gabapentin, lamotrigine, and topiramate. In addition, cell cultures were also supplemented with the classic osteoclastogenic promoters M-CSF $(25 \mathrm{ng} / \mathrm{ml})$ and RANKL $(40 \mathrm{ng} / \mathrm{ml}$; Costa-Rodrigues, Martins, \& Fernandes, 2012). Both the AED and the M-CSF and RANKL were renewed at each medium change. Cultures were assessed for the tartrate-resistant acid phosphatase (TRAP) activity, the presence of TRAP + multinucleated cells, apoptosis and the presence of actin rings, vitronectin receptor (VNR), and calcitonin receptor (CTR), at days 7,14 , and 21 .

Cultures treated with the minimum tested concentration of each AED that elicited a significant effect in osteoclastic behavior (valproic acid, gabapentin, and lamotrigine: $10^{-7} \mathrm{M}$; carbamazepine and topiramate: $10^{-6} \mathrm{M}$ ), were characterized for the expression of osteoclastic genes, calcium phosphate resorbing ability, and for the involvement of several intracellular pathways in cell response. Cultures were assessed at days 7, 14, and 21.

\section{2 | Osteoblastic cell cultures}

Human mesenchymal stem cells derived from bone marrow (HMSC, Innoprot, Bizkaia, Spain), were used as osteoblastic precursors. HMSC were maintained in $\alpha$-MEM supplemented with $10 \%$ fetal bovine serum (FBS), $100 \mathrm{IU} / \mathrm{ml}$ penicillin, $2.5 \mathrm{\mu g} / \mathrm{ml}$ streptomycin, $2.5 \mu \mathrm{g} / \mathrm{ml}$ amphotericin $\mathrm{B}$, and $50 \mu \mathrm{g} / \mathrm{ml}$ ascorbic acid. After reaching $~ 70-80 \%$ confluence, cells were detached with $0.05 \%$ trypsin $+0.5 \mathrm{mM}$ EDTA and seeded at $3.3 \times 10^{3}$ cells $/ \mathrm{cm}^{2}$. Cell cultures were performed for 21 days in the same culture medium, being incubated in a $5 \% \mathrm{CO}_{2}$ humidified atmosphere at $37^{\circ} \mathrm{C}$. Culture medium was replaced once a week.

Cell cultures were exposed to a wide range of the different tested AED $\left(10^{-8}-10^{-4} \mathrm{M}\right), 24 \mathrm{hr}$ after cell seeding, in the presence of the osteogenic inducer dexamethasone $(10 \mathrm{nM}$; Costa-Rodrigues, Reis, Castro, \& Fernandes, 2016a). The tested AED were valproic acid, carbamazepine, gabapentin, lamotrigine, and topiramate. Both the AED and the dexamethasone were renewed at each medium change. Cultures were assessed for the alkaline phosphatase (ALP) activity, cell viability, apoptosis, and cellular morphology at days 7, 14, and 21.

Cultures treated with the minimum tested concentration of each AED that elicited a significant effect in osteoblastic behavior (valproic acid, carbamazepine, and lamotrigine: $10^{-6} \mathrm{M}$; gabapentin and topiramate: $10^{-7} \mathrm{M}$ ), were further assessed for expression of osteoblastic genes and for the involvement of several intracellular pathways in cell response. Cultures were assessed at days 7, 14, and 21. 


\section{3 | Characterization of cellular responses}

\subsection{1 | Tartrate-resistant acid phosphatase (TRAP) and alkaline phosphatase (ALP) activities}

TRAP and ALP activities were determined by the para-nitrophenylphosphate ( $p N P P$ ) hydrolysis assay. For that, after being washed two times with PBS, cells were solubilized with $0.1 \%$ (V/V) Triton X-100. For the TRAP activity quantification, cellular extracts were incubated $\left(1 \mathrm{hr}, 37^{\circ} \mathrm{C}\right.$ ) with $12.5 \mathrm{mM} \mathrm{pNPP}$ prepared in $0.04 \mathrm{M}$ tartaric acid and $0.09 \mathrm{M}$ citrate $(\mathrm{pH} 4.8)$; for the ALP activity analysis, cellular extracts were incubated $\left(30 \mathrm{~min}, 37^{\circ} \mathrm{C}\right.$ ) with $12.5 \mathrm{mM}$ pNPP in alkaline buffer solution, $\mathrm{pH}$ 10.3. In both methods, after addition of $5 \mathrm{M} \mathrm{NaOH}$, the absorbance of the samples was measured at $400 \mathrm{~nm}$ in an ELISA plate reader (Synergy HT, Biotek, Winooski, Vermont, EUA). TRAP and ALP activities were normalized with total protein content quantified by the Bradford's method (Bradford, 1976), and results are expressed as $\mathrm{nmol} / \mathrm{min} \mu \mathrm{g}_{\text {protein }}{ }^{-1}$.

\subsection{2 | TRAP-positive multinucleated cells}

PBMC cultures were washed two times with PBS and fixed for $10 \mathrm{~min}$ with 3.7\% formaldehyde. Following, cells were stained for TRAP with acid phosphatase, leukocyte (TRAP) kit (Sigma, MO), according to manufacturer's instructions. For that, cells were incubated with $0.12 \mathrm{mg} / \mathrm{ml}$ naphtol hAS-BI, in the presence of $6.76 \mathrm{mM}$ tartrate and $0.14 \mathrm{mg} / \mathrm{ml}$ Fast Garnet GBC, for $1 \mathrm{hr}$ at $37^{\circ} \mathrm{C}$, in the dark. After incubation, cell layers were washed, stained with hematoxylin and TRAP-positive multinucleated cells were counted under a phase contrast microscope (Nikon TMS, Tokyo, Japan).

\subsection{3 | Calcium staining}

HMSC cultures were washed two times with PBS and fixed with $3.7 \%$ formaldehyde for $10 \mathrm{~min}$. After incubation, cell layers were covered with a $1 \%$ alizarin sodium solution $\left(0.028 \%\right.$ in $\left.\mathrm{NH}_{4} \mathrm{OH}\right), \mathrm{pH} 6.4,2$ min, and then rinsed with distilled water and acid ethanol (ethanol, $0.01 \%$ $\mathrm{HCL}$ ). Calcium deposits stained red.

\subsubsection{Cell viability}

Cell viability was assessed by the MTT assay, which is based on the reduction of the tetrazolium salt MTT (3-[4,5-dimethylthiazol-2-yl]2,5-diphenyltetrazolium bromide; $0.5 \mathrm{mg} / \mathrm{mL}$ ), by viable cells to form a purple formazan product after $3 \mathrm{hr}$ of incubation. After solubilization in dimethyl sulfoxide (DMSO), the absorbance of the samples was measured at $545 \mathrm{~nm}$ on a microplate reader (Synergy HT, Biotek).

\subsection{5 | Apoptosis quantification}

Apoptosis was assessed by the caspase- 3 activity quantification. Briefly, cells were washed two times with PBS and evaluated for the caspase-3 activity with EnzCheck ${ }^{\circledR}$ Caspase-3 Assay Kit \#2 (Molecular Probes, Eugene), according to manufacturer's instructions. Fluorescence was measured at 496/520 nm (excitation/emission) in an ELISA plate reader (Synergy HT, Biotek). Results obtained in each experimental condition were normalized with the value obtained in the corresponding control (absence of AED) and were presented as a \% of response compared with the control.

\subsubsection{Actin, vitronectin receptor (VNR), and calcitonin receptor (CTR) staining}

Cell cultures were washed with PBS and fixed with 3.7\% formaldehyde, for $10 \mathrm{~min}$ at room temperature. After that, cells were permeabilized with $0.1 \%$ Triton X-100, for $5 \mathrm{~min}$, and stained for actin with $5 \mathrm{U} / \mathrm{ml}$ Alexa Fluor1 647-Phalloidin (Invitrogen, Carlsbad, California, EUA), and, in the case of PBMC cultures, also for vitronectin and calcitonin receptors (VNR and CTR) with $50 \mathrm{mg} / \mathrm{ml}$ mouse $\mathrm{lgGs}$ anti-VNR and IgGs anti-CTR (R\&D Systems, Minneapolis, EUA), respectively. Detection of IgGs anti-VNR and IgGs anti-CTR was conducted with $2 \mathrm{mg} / \mathrm{ml}$ Alexa Fluor1 488-Goat antimouse IgGs. Cells were observed under confocal laser scanning microscopy (CLSM).

\subsection{7 | Osteoclast and osteoblast gene expression}

Gene expression was evaluated by quantitative real-time polymerase chain reaction ( $q P C R$ ). The tested housekeeping genes were betaglucuronidase (GUSB) and proteasome subunit beta type-6 (PSMB6). The analyzed osteoclast-related genes were c-myc, c-src, TRAP, cathepsin $\mathrm{K}$ (CATK), and carbonic anhydrase 2 (CA2; Costa-Rodrigues et al., 2014) and the osteoblast-related genes were collagen type 1 (COL1), ALP, and bone morphogenetic protein 2 (BMP2; CostaRodrigues et al., 2016a). RNA was isolated with a RNeasy ${ }^{\circledR}$ Mini Kit (QIAGEN, Hilden, Germany) according to manufacturer's instructions. Reverse transcription complementary DNA (cDNA) synthesis was performed with the DyNAmo cDNA synthesis kit (Finnzymes, Finland) and random hexamers according to the manufacturer's instructions. Following, cDNA samples ( $2 \mathrm{ng}$ ) were amplified with a DyNAmo Flash SYBR green qPCR kit (Finnzymes) on a Rotor-Gene thermocycler (Qiagen). The annealing temperature was $55^{\circ} \mathrm{C}$ and the extension time was $15 \mathrm{~s}$. The primers used are listed in Table 1. The values obtained were normalized with the results obtained for the two tested housekeeping genes.

\subsection{8 | Calcium phosphate resorbing ability}

For resorbing ability assay, PBMC cultures were performed on calcium phosphate coated culture plates (BD BioCoat ${ }^{\mathrm{TM}}$ Osteologic $^{\mathrm{TM}}$ Bone Cell Culture Plates, BD Biosciences, NJ). Cells were maintained for 21 days in the absence or presence of AED. At the end of the culture period, cells were bleached with $6 \% \mathrm{NaOCl}$ and $5.2 \% \mathrm{NaCl}$, and the remaining calcium phosphate layers were visualized under phase contrast light microscopy (Nikon TMS, Tokyo, Japan). Resorption lacunae were identified and total resorbed area was quantified with ImageJ 1.41 software. Results are presented as a \% of resorbed area. 
TABLE 1 Primers used on qPCR analysis of PBMC and HMSC cultures

\begin{tabular}{|c|c|c|}
\hline Gene & 5' Primer & 3' Primer \\
\hline GUSB & 5'-TGCAGCGGTCTGTACTTCTG-3' & 5'-CCTTGACAGAGATCTGGTAATTCA-3' \\
\hline PSMB6 & 5'-GCCGGCTACCTTACTACCTG-3' & 5'-AAACTGCACGGCCATGATA-3' \\
\hline$c$-src & 5'-AAGCTGTTCGGAGGCTTCAA-3' & 5'-TTGGAGTAGTAGGCCACCAG-3' \\
\hline TRAP & 5'-ACCATGACCACCTTGGCAATGTCTC-3' & 5'-ATAGTGGAAGCGCAGATAGCCGTT-3' \\
\hline CA2 & 5'-GGACCTGAGCACTGGCATAAGGACT-3' & 5'-AAGGAGGCCACGAGGATCGAAGTT-3' \\
\hline COL1 & 5'-TCCGGCTCCTGCTCCTCTTA-3' & 5'-ACCAGCAGGACCAGCATCTC-3' \\
\hline$A L P$ & 5'-ACGTGGCTAAGAATGTCATC-3' & 5'-CTGGTAGGCGATGTCCTTA-3' \\
\hline BMP-2 & 5'-GACGAGGTCCTGAGCGAGTT-3' & 5'-GCAATGGCCTTATCTGTGAC-3' \\
\hline
\end{tabular}

Note. HMSC: human mesenchymal stem cells; PBMC: human peripheral blood mononuclear cells; q-PCR: quantitative real-time polymerase chain reaction.

\subsection{9 | Intracellular signaling mechanisms}

The ability of AED to modulate several common signaling pathways involved either in osteoclastogenesis (Costa-Rodrigues et al., 2016b; Soysa \& Alles, 2016) and osteoblastogenesis (Costa-Rodrigues et al., 2016a; Valenti et al., 2016) was assessed. For that, PBMC and HMSC cultures were treated with the minimum AED concentration that elicited a significant response on each cell type, and simultaneously, with commercial signaling pathway inhibitors (the AED and the inhibitors were renewed at each medium change). The tested pathways were: MEK (inhibitor U0126, $1 \mu \mathrm{M}$ ), NFkB (inhibitor PDTC, $10 \mu \mathrm{M}$ ), PKC (inhibitor G06983, $5 \mu \mathrm{M}$ ), and JNK (inhibitor SP600125, $10 \mu \mathrm{M}$ ). Cultures were assessed for TRAP (PBMC cultures) and ALP (HMSC cultures) activities.

\section{4 | Statistical analysis}

Data presented in this study are the means of three independent experiments. Statistical differences were assessed using an unpaired Student's t-test with Bonferroni correction for multiple comparisons. All data are presented as the mean \pm standard deviation/error. For values of $p \leq .05$, differences were considered statistically significant.

\section{3 | RESULTS}

\section{1 | Effects of AED on osteoclastogenesis}

It was observed that valproic acid, at low doses $\left(10^{-7} \mathrm{M}\right)$, promoted an increase in the TRAP activity, being $29 \%$ higher than the control (Figure 1a). At higher doses, it elicited a dose-dependent decrease in cell response. The other tested AED caused a dose-dependent decrease in that parameter, which became significant at $10^{-7} \mathrm{M}(\sim 20 \%$ and $\sim 25 \%$ lower for gabapentin and lamotrigine, respectively) and $10^{-6} \mathrm{M}(\sim 17 \%$ and $\sim 28 \%$ lower for carbamazepine and topiramate, respectively). A similar pattern of response was observed when the amount of osteoclastic cells (TRAP + multinucleated cells) in each experimental condition was quantified (Figure 1b), that means, valproic acid was the only $A E D$ that increased cell response (at $10^{-7} \mathrm{M}$ ), whereas higher concentration, and the other AED promoted a dose-dependent decrease. To analyze if the decrease in the amount of osteoclastic cells promoted by the AED was due to an increase in cell death, the apoptotic response was also evaluated (Figure 1c). It was observed that all the tested AED, at low concentrations $\left(10^{-8} \mathrm{M}\right)$ did not affect the caspase-3 activity, whereas at $10^{-6} \mathrm{M}$ only gabapentin and lamotrigine significantly increased it $\left(\sim 31 \%\right.$ and $\sim 36 \%$, respectively). At $10^{-4} \mathrm{M}$, all the AED caused a significant increase in apoptosis.

In all tested conditions it was possible to observe cells with distinctive osteoclastic features namely, actin rings and expression of VNR and CTR (Figure 2a). PBMC cultures treated with the minimum concentration of each AED that caused a significant effect in the TRAP activity were further characterized for additional parameters. Regarding gene expression (Figure $2 \mathrm{~b}$ ), it was observed that valproic acid was able to stimulate the expression of all analyzed genes, namely, the osteoclastogenic factors c-myc and c-src and the functional markers TRAP, CATK, and CA2 ( 17-49\% higher than the control). On the other hand, all the other AED elicited a significant decrease of gene expression $(\sim 16-47 \%$ lower than the control). Calcium phosphate resorbing ability was also modulated by the AED (Figure 2c). Once again, valproic acid was the only drug that caused a significant increase in cell response $(\sim 71 \%)$, whereas the other AED elicited a decrease in the resorbing ability ( 26-50\%).

Having established how the different AED affected osteoclast differentiation and function, the involved intracellular mechanisms were characterized (Figure 3). In the absence of any AED, PDTC completely abolished the TRAP activity, whereas the remaining inhibitors caused a partial inhibition in cell response. In the presence of valproic acid, U0126 did not affect the TRAP activity, and SP600125 promoted a sharp decrease on it. Carbamazepine treatment elicited a higher inhibition in the presence of U0126 and GO6983, whereas SP600125 caused only a slight decrease in the TRAP activity. In the presence of gabapentin U0126 did not affect cell response, and in the presence of lamotrigine, it was G06983 that did not change the TRAP activity. Finally, following supplementation with topiramate, PBMC cultures exhibited a lower response in the presence of U0126, and in the presence of SP600125 TRAP activity was completely abolished. 

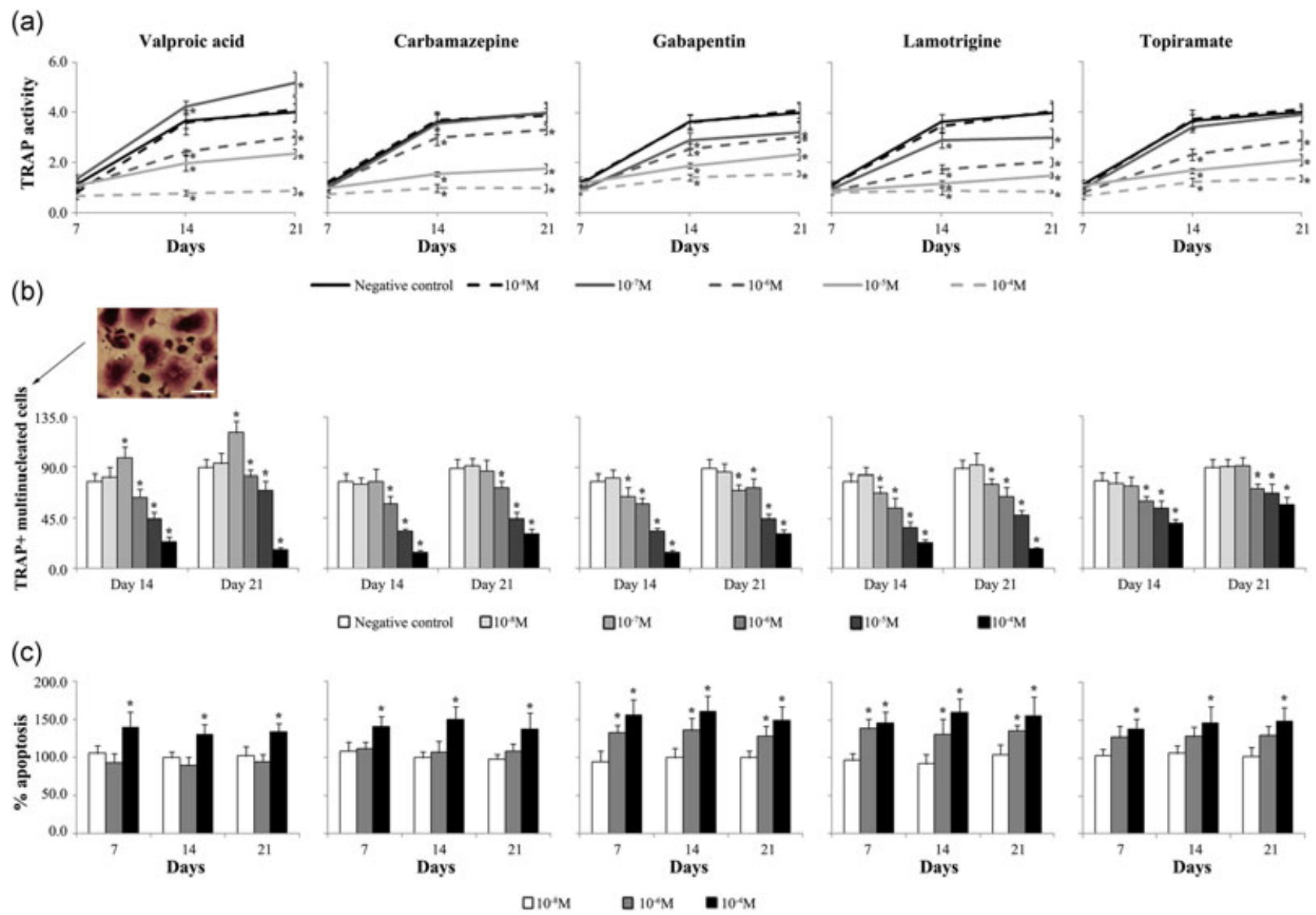

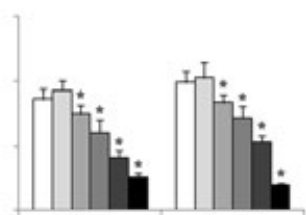

Day 14

Day 21

口10\% $\mathrm{M}$

$10^{-4} \mathrm{M}$

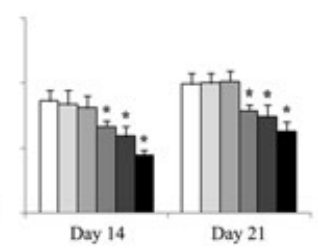

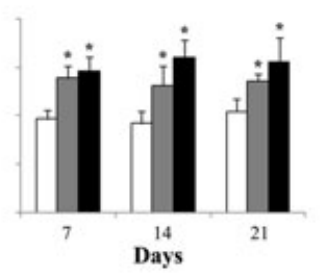

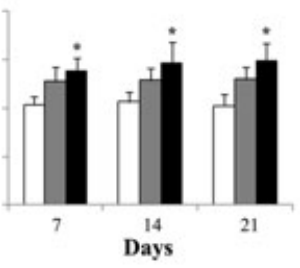

FIGURE 1 Dose-response curves of PBMC cultures treated with different AED (valproic acid, carbamazepine, gabapentin, lamotrigine, and topiramate). Tested concentrations: $10^{-8} \mathrm{M}-10^{-4} \mathrm{M}$. Cellular behavior was assessed at days 7,14 , and 21 . Cell cultures performed in the absence of any AED were used as negative control. (a) The TRAP activity, assessed by pNPP hydrolysis method. (b) TRAP + multinucleated cells quantification by optic microscopy following staining). White bar represents $100 \mu \mathrm{m}$. (c) Caspase-3-activity assessed by fluorescence quantification. ${ }^{*}$ Significantly different from the control. AED: antiepileptic drugs; PBMC: human peripheral blood mononuclear cells; TRAP: tartrate-resistant acid phosphatase [Color figure can be viewed at wileyonlinelibrary.com]

\section{2 | Effects of AED on osteoblastogenesis}

At low doses, valproic acid and topiramate elicited a significant increase in the ALP activity, being $\sim 23 \%$ with $10^{-6} \mathrm{M}$ valproic acid, and $\sim 14 \%$ with $10^{-7} \mathrm{M}$ topiramate (Figure 4a). At higher concentrations, both AED caused a decrease in cell response. The other AED promoted a dosedependent inhibition of the ALP activity, which became significant at $10^{-7} \mathrm{M}\left(\sim 12 \%\right.$ for gabapentin) and at $10^{-6} \mathrm{M}(\sim 12 \%$ and $\sim 14 \%$ for carbamazepine and lamotrigine, respectively). At $10^{-6} \mathrm{M}$, valproic acid (a)

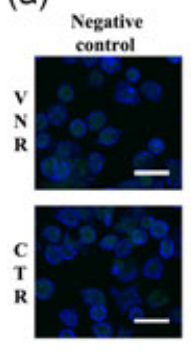

(b)

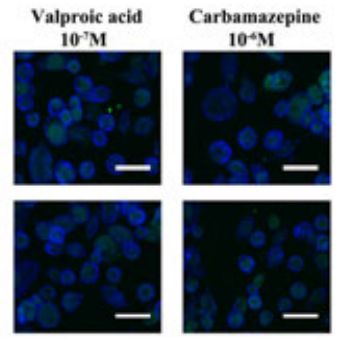

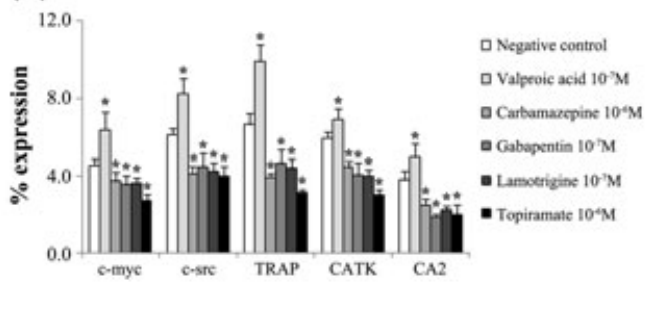

(c)

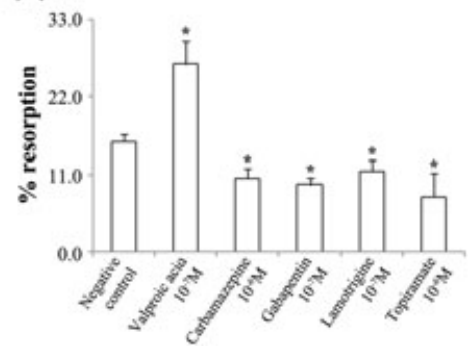

FIGURE 2 Effects of AED (valproic acid, carbamazepine, gabapentin, lamotrigine, and topiramate) on PBMC cultures. (a) Representative images of confocal microscopy showing the presence of cells with actin rings (blue) and expressing vitronectin and calcitonin receptors (VNR and CTR, respectively) (green); white bars represent $220 \mu \mathrm{m}$. (b) Expression of osteoclast-related genes, namely, the osteoclastogenic factors c-myc and c-src and the osteoclastic functional genes TRAP, CATK, and CA2. (c) Calcium phosphate resorbing ability after 21 days of culture in the presence of the tested AED. *Significantly different from the control. AED: antiepileptic drugs; PBMC: human peripheral blood mononuclear cells [Color figure can be viewed at wileyonlinelibrary.com] 

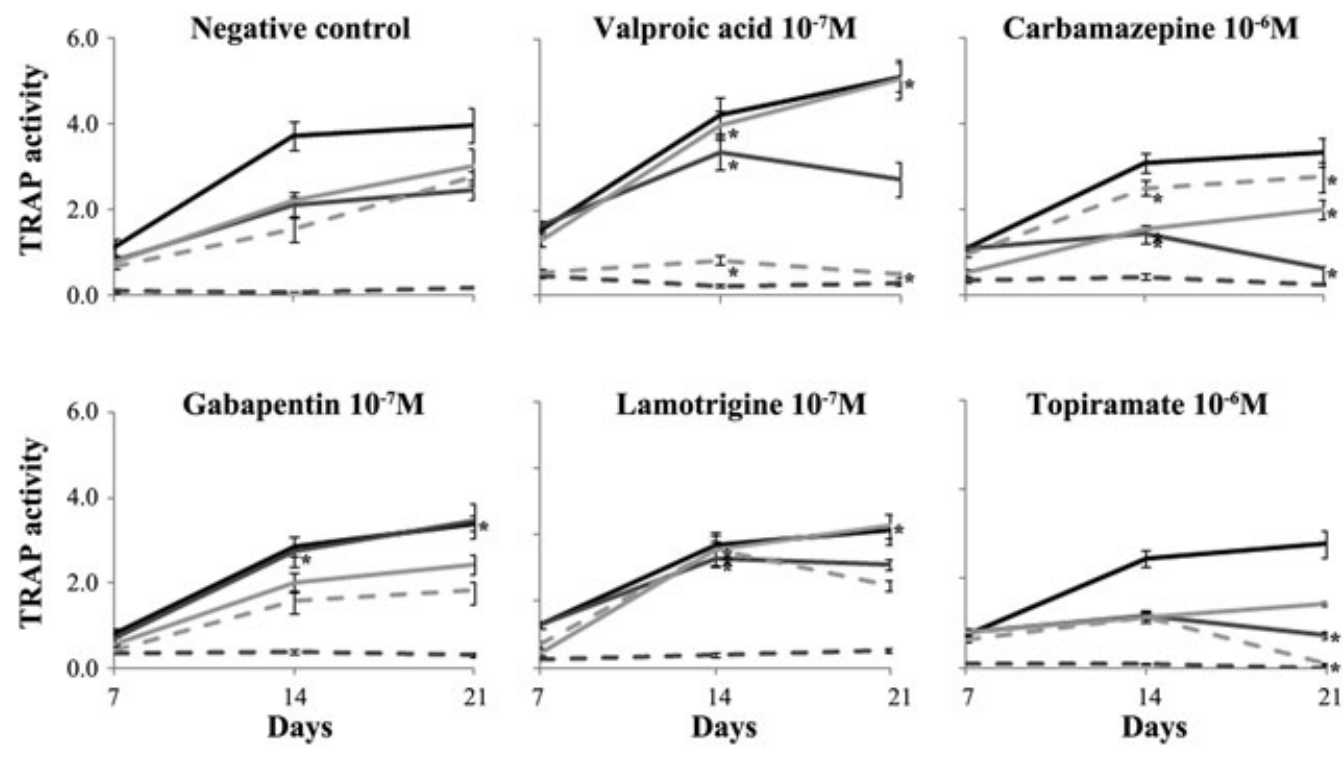

$\longrightarrow$ No inhibitor $\quad-\quad 01261 \mu \mathrm{M}$

- PDTC $10 \mu \mathrm{M}$

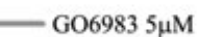

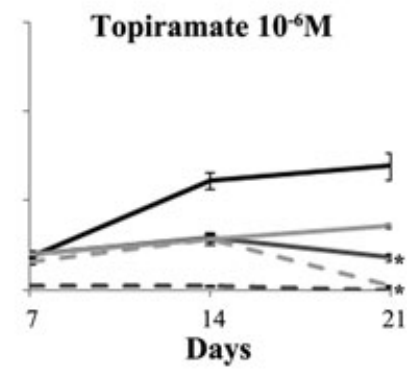

FIGURE 3 Modulation of MEK, NFkB, PKC, and JNK signaling pathways by AED, in PBMC cultures. The tested concentration of each AED was selected according to the dose-response curves performed for each drug. Cell response was evaluated by the TRAP activity. *Significantly different from the control. AED: antiepileptic drugs; PBMC: human peripheral blood mononuclear cells; TRAP: tartrate-resistant acid phosphatase

and topiramate promote an increase in calcium deposits, whereas the remaining drugs elicited an opposite response (Figure $4 \mathrm{~b}$ ). Regarding cell viability (Figure 4c), all the tested AED caused a dose-dependent decrease, which was significant at $10^{-6} \mathrm{M}(\sim 16 \%, \sim 17 \%$ and $\sim 14 \%$ for valproic acid, carbamazepine, and topiramate, respectively) and $10^{-5} \mathrm{M}$
( $10 \%$ and $\sim 14 \%$ for gabapentin and lamotrigine, respectively). At $10^{-8} \mathrm{M}$ none of the tested AED affected apoptosis (Figure $4 \mathrm{~d}$ ), and at $10^{-6} \mathrm{M}$ only valproic acid and topiramate promoted a significant increase ( $44 \%$ and $\sim 29 \%$, respectively). All tested AED, at $10^{-4} \mathrm{M}$, prompted an increase in the caspase- 3 activity $(\sim 45-80 \%)$.
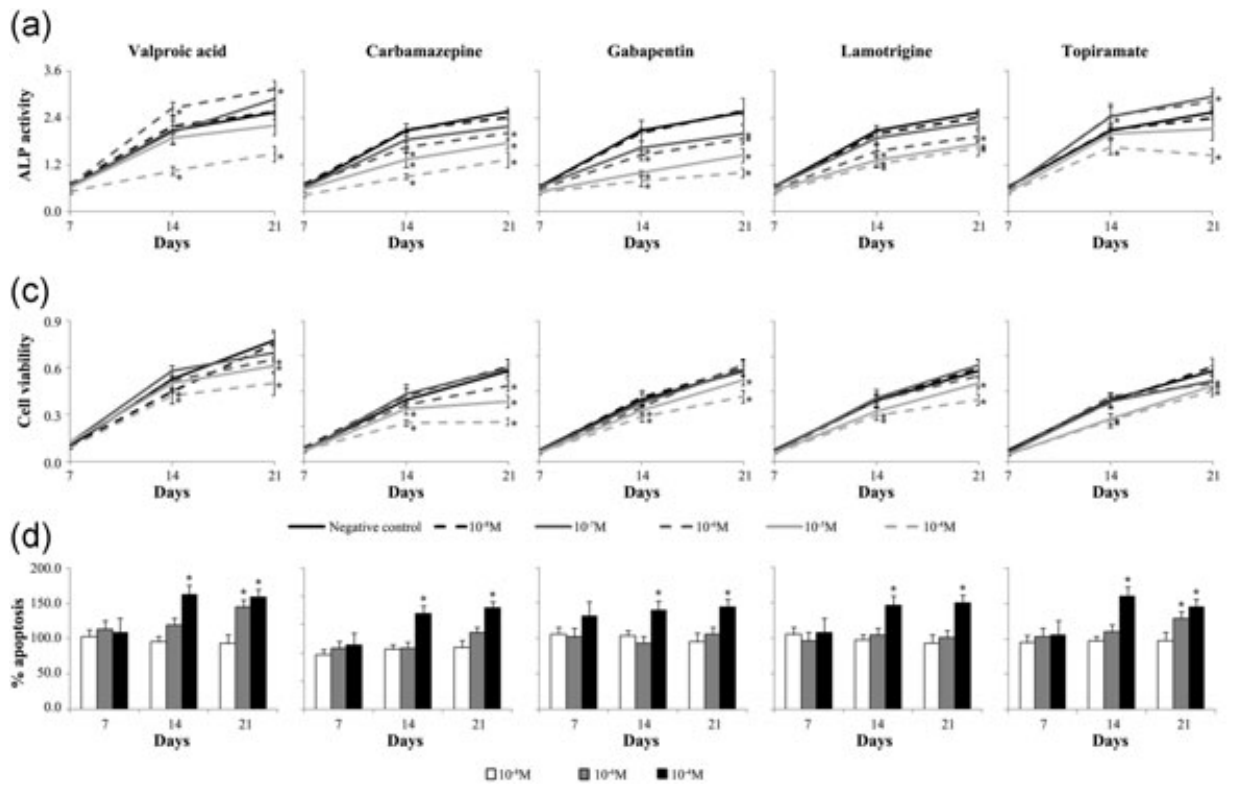

Day

(b)
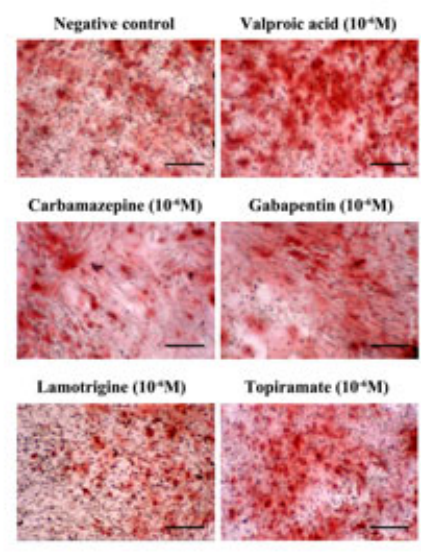

FIGURE 4 Dose-response curves of HMSC cultures treated with different AED (valproic acid, carbamazepine, gabapentin, lamotrigine, and topiramate). Tested concentrations: $10^{-8} \mathrm{M}-10^{-4} \mathrm{M}$. Cellular behavior was assessed at days 7, 14, and 21. Cell cultures performed in the absence of any AED were used as negative control. (a) The ALP activity, assessed by the $p$ NPP hydrolysis method. (b) Representative images of HMSC after Alizarin Red staining. Black bars represent $120 \mu \mathrm{m}$. (c) Cell viability, assessed by the MTT assay. (d) The Caspase-3-activity assessed by fluorescence quantification. *Significantly different from the control. AED: antiepileptic drugs; HMSC: human mesenchymal stem cells; MTT: 3-(4,5-dimethylthiazol-2-yl)-2,5-diphenyltetrazolium bromide [Color figure can be viewed at wileyonlinelibrary.com] 
(a)

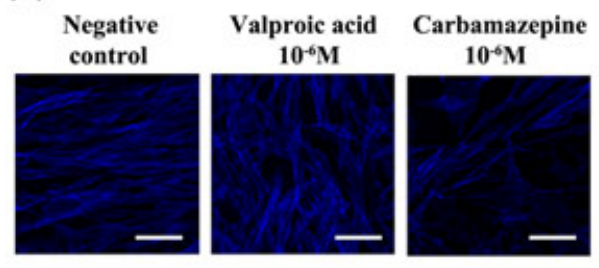

(b)

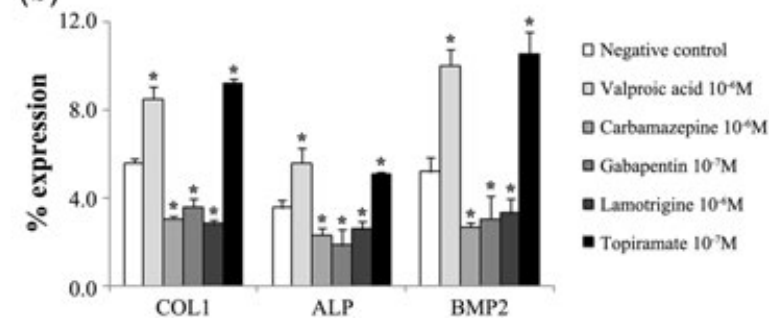

FIGURE 5 Effects of AED (valproic acid, carbamazepine, gabapentin, lamotrigine, and topiramate) on PBMC cultures. (a) Representative images of confocal microscopy showing the presence of cells stained for actin (blue); white bars represent $60 \mu \mathrm{m}$. (b) Expression of osteoblastrelated genes, namely, the functional genes COL1, ALP, and BMP2. *Significantly different from the control. AED: antiepileptic drugs; PBMC: human peripheral blood mononuclear cells [Color figure can be viewed at wileyonlinelibrary.com]

In all tested conditions, cells were well-spread and exhibited a normal morphology, with distinctive cell-to-cell contacts between them (Figure 5a). Regarding gene expression (Figure 5b), valproic acid and topiramate elicited a significant increase on the expression of the functional genes COL1, ALP, and BMP2 ( 52-98\%), whereas the remaining three $A E D$ caused a decrease in the expression of the analyzed genes ( 27-49\%).

After the characterization of the effects of the different AED on osteoblast differentiation, the associated intracellular mechanisms were analyzed (Figure 6). It was observed that, in negative control condition (absence of any AED), all the used inhibitors promoted a partial decrease in the ALP activity, although G06983-related inhibition did not reach significance. In the presence of valproic acid, U0126 and G06983 caused a lower decrease in cell response; on the other hand SP600125 caused a sharper decrease than in the negative control. Cultures performed in the presence of carbamazepine were not significantly affected by U0126, whereas G06983 displayed an increase in its inhibitory effect. Supplementation with gabapentin promoted a lower decrease of the ALP activity in the presence of SP600125, and an absence of inhibition in the presence of PDTC; G06983 caused a higher inhibition, compared with the negative control. Cell cultures performed with topiramate were not significantly affected by U0126 and SP600125.

\section{4 | DISCUSSION}

AED are a class of drugs used worldwide, not only for the treatment of epilepsy, but also in cases of convulsions, seizures, pain, neuropathy, and psychiatric disorders (Fan et al., 2016; Hant \& Bolster, 2016; Pitetzis et al., 2017). They present many potential side effects, including dizziness, drowsiness, mental slowing, skin rashes, hepatotoxicity, pancreatic insufficiency, behavioral disorders, increased risk of metabolic syndrome, and nephrolithiasis (Fan et al., 2016; Lin et al., 2016). Also, disruption of bone metabolism is described for decades, although the exact consequences in bone
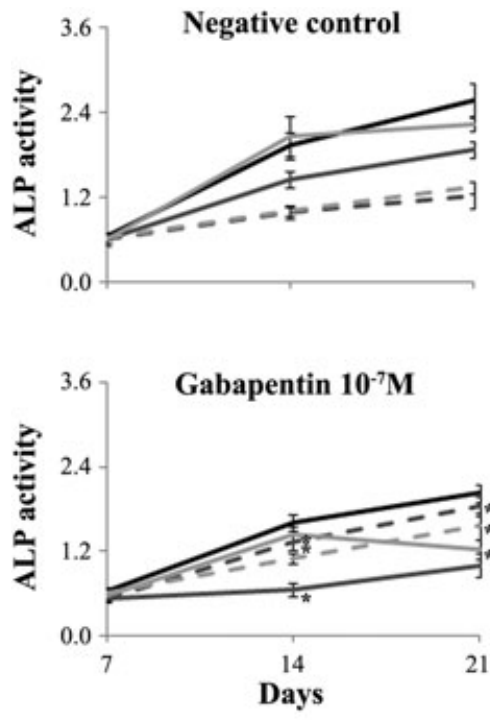

No inhibitor

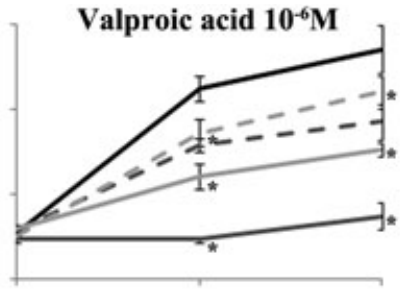

Lamotrigine $10^{-6} \mathrm{M}$

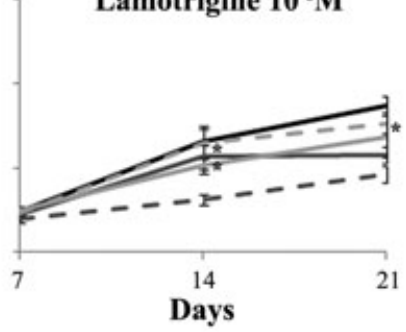

- PDTC $10 \mu \mathrm{M}$

\section{Carbamazepine $10^{-6} \mathrm{M}$}

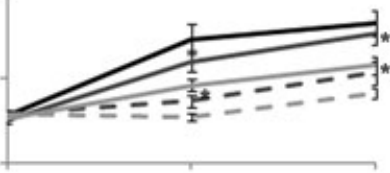

Topiramate $10^{-7} \mathbf{M}$

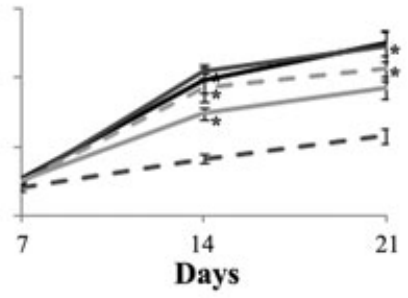

- SP600125 $10 \mu \mathrm{M}$

FIGURE 6 Modulation of MEK, NFkB, PKC, and JNK signaling pathways by AED, in HMSC cultures. The tested concentration of each AED was selected according to the dose-response curves performed for each drug. Cell response was evaluated by the ALP activity. ${ }^{*}$ Significantly different from the control. AED: antiepileptic drugs; ALP: alkaline phosphatase; HMSC: human mesenchymal stem cells 
tissue remain elusive. In this study, the effects of five different AED on human primary bone cells (osteoclasts and osteoblasts) were characterized.

The tested AED were the valproic acid, carbamazepine, gabapentin, lamotrigine, and topiramate. The first two are classical AED, which are members of the first generation of this class, whereas gabapentin, lamotrigine, and topiramate belong to the newer class of AED, being apparently safer and better tolerated by patients (Fan et al., 2016). The AED were selected expecting to include molecules with different mechanisms of action, and the tested concentrations were chosen to include the plasma concentration achieved following a common therapeutic usage of each drug (Abou-Khalil, 2005; Haroutiunian et al., 2009; O'Dougherty et al., 1987; Popov et al., 2013; Rose \& Kam, 2002). However, it is important to highlight that those values may not represent the exact concentrations present in bone tissue, because the vascularization and the particular characteristics of its extracellular matrix contribute to a specific biochemical microenvironment in that tissue.

Valproic acid is short-chain fatty acid with a branched structure, derived from valeric acid. It displays antiepileptic properties, being also prescribed in other pathological conditions, such as migraine and bipolar disease (Pitetzis et al., 2017). It has some important cellular effects, namely, inhibition of cytochrome P450, promotion of histone acetylation, and modulation of several cellular functions, differentiation, and apoptosis (Pitetzis et al., 2017). Regarding its side effects, valproic acid is by far the better studied AED, being associated to hepatotoxicity, pancreatitis, teratogenicity, and an increased risk to develop metabolic syndrome. In addition, there are many reports claiming that valproic acid is linked to a significant decrease in bone mineral density and to an increase in bone fracture risk (Albaghdadi, Alhalabi, Alourfi, \& Youssef, 2016; Ecevit et al., 2004; GniatkowskaNowakowska, 2010; Guo et al., 2001; Hamed, Moussa, Youssef, Abd ElHameed, \& NasrEldin, 2014; Lee, Lyles, \& Colon-Emeric, 2010; Tsukahara et al., 2002; Zhang, Zheng, Zhu, Zhang, \& Zheng, 2015). It has also been suggested that valproic acid delays growth velocity (Lee et al., 2013; Lin et al., 2016). Nevertheless, other studies failed to prove any relationship between valproic acid consumption and disturbances in bone metabolism (Elliott, Jacobson, \& Haneef, 2007; Kim, Lee, Choi, Chung, \& Lee, 2007; Serin, Koc, Temelli, \& Esen, 2015; Shen et al., 2014; Triantafyllou et al., 2010). Regarding bone turnover markers, there are also conflicting data, stating that valproic acid increases (Kim et al., 2007; Oner et al., 2004; Verrotti, Agostinelli, Coppola, Parisi, \& Chiarelli, 2010), decreases (Song et al., 2005; Tsukahara et al., 2002) or does not affect osteocalcin serum levels. In more detail, ALP levels seem to be increased by this drug (Lin et al., 2016; Verrotti et al., 2010), as well as bone resorption markers, such as ionized plasma calcium, carboxyterminal telopeptide of type I collagen (Sato et al., 2001), and the plasma TRAP activity (Lin et al., 2016; Rauchenzauner et al., 2010). On the other hand, in a study with female children and adolescents, valproic acid appeared to promote a decrease in serum TRAP levels (Lin et al., 2016).

Carbamazepine is an iminodibenzyl derivative that belongs to the conventional enzyme-inducing antiepileptic drugs (AED), which displays the ability to induce hepatic cytochrome P450 function (Pitetzis et al., 2017). It is widely used for the treatment of partial and secondary generalized seizures (Mantegazza, Curia, Biagini, Ragsdale, \& Avoli, 2010). Besides nausea, vomiting, weight gain, and other common side effects, carbamazepine has been also associated with bone loss and increased risk of fracture (Jette et al., 2011), though there are also reports that state an increase in bone formation markers (Verrotti, Greco, Latini, Morgese, \& Chiarelli, 2002).

Gabapentin presents a chemical structure with similarities with GABA (Fan et al., 2016). It has a high lipophilicity, being effective in the treatment of headaches, inflammatory pain, and several neuropathy-associated pains (Rose \& Kam, 2002). It presents as the most relevant side effects, sexual dysfunction, weight gain, dizziness, fatigue, and bone loss and increased risk of fracture (Andress et al., 2002; Jette et al., 2011).

Lamotrigine is a newer non-enzyme-inducer AED that is effective in the treatment of clonic-tonic and partial seizures (Fan et al., 2016). Its main side effects include headache, dizziness, sedation, insomnia, and ataxia. Furthermore, it has been linked to bone loss, disturbed child growth and decreased bone mineral density (Andress et al., 2002; Guo et al., 2001), although this effect seems so be particularly relevant when lamotrigine is administered in combination with valproic acid (Guo et al., 2001). However, there are also studies that have failed to demonstrate a strong relationship between lamotrigine and bone metabolic imbalances (Kim et al., 2007).

Topiramate is a derivative of fructose (Fan et al., 2016). It is a non-enzyme-inducer AED approved as an adjuvant for primary clonic-tonic and partial seizures, as well as a prophylactic agent in migraines, depression, bipolar disorder, and among others (LysengWilliamson \& Yang, 2007). Its most common adverse effects are somnolence, paresthesia, poor concentration, and weight loss (Fan et al., 2016; Lyseng-Williamson \& Yang, 2007). In addition, topiramate has also been linked to disturbances in bone metabolism, namely, abnormal BMD (Ali, Herial, Orris, Horrigan, \& Tietjen, 2011; Coppola et al., 2009). One possible explanation for these effects is the fact that this molecule inhibits carbonic anhydrase, which can promote metabolic acidosis and, consequently, can lead to a decrease in mineral content of bone. However, one cannot exclude other mechanisms of action neither a potential direct effect of the drug in bone cells, though there are no reports about this issue.

Globally, the effects of AED in bone metabolism still controversial, but growing evidence points to imbalances in bone turnover and an increased risk of fracture (Kim et al., 2007; Pitetzis et al., 2017; Tsukahara et al., 2002). On one hand, the risk of fracture seems to be higher in the case of P450-modulator drugs, such as valproic acid or carbamazepine (Hant \& Bolster, 2016). Despite the consequences on bone tissue, the AED modulation of bone metabolism may occur by different ways. They may exert their effects on bone tissue via indirect mechanisms, such as stimulation of vitamin D catabolism and, consequently, a decrease on intestinal calcium absorption and parathyroid hormone secretion (Pitetzis et al., 2017). However, it is also possible that at least some of them may have direct actions on the differentiation and function of bone cells, particularly at 
osteoclast and osteoblast level, an issue that is extremely poor documented.

In the present work, it was observed that all the tested AED had the ability to affect human osteoclastogenesis. Among the five AED, only valproic acid was able to stimulate the process, at low doses. These effects were related not only to the differentiation process, but also to the resorbing function of mature osteoclasts. At least partially, some of the results may be related to an increase in apoptosis, because at high doses all the tested AED stimulated the process. The different AED also caused differential effects on the expression of different genes involved in the osteoclast development and function (c-myc, c-src, TRAP, CATK, and CA2), that were dependent of the dose of each AED. Globally the pattern of response was similar to that observed in the TRAP activity. Although in some experimental conditions the magnitude of the response was somehow low, it is important to note that any increase in gene transcription may be associated with significant changes in the production of the corresponding protein, because each mRNA can be translated multiple times. The AED also affected the resorbing function of osteoclasts. Once again, valproic acid was able to elicit an increase in cell response, whereas the remaining AED promoted an inhibition of cell response. Regarding the involved intracellular mechanisms, the results pointed to differential effects caused by the different drugs, which is not surprising, because although they belong to the same pharmacological family, they are chemically very different and, thus, present different mechanisms of action. The MEK pathway appeared to be more relevant in the presence of carbamazepine and topiramate, but not important in the presence of gabapentin. Valproic acid and lamotrigine elicited a downregulation of PKC, whereas carbamazepine downregulated it. JNK was critical for osteoclastogenesis following supplementation with topiramate, whereas an opposite situation was observed with carbamazepine. To have a more detailed perspective about the specific mechanisms affected by each AED, the analysis of the expression and, in some cases, phosphorylation/dephosphorylation ratio of different intracellular molecules involved in important signaling pathways, should be conducted. This study is underway.

Osteoblastogenesis was stimulated by low doses of valproic acid and topiramate, whereas the remaining AED promoted a dosedependent decrease of the process. A similar behavior was observed regarding the presence of calcium deposits, assessed by alizarin red staining. The observed stimulatory effects were not due to an increase in cell viability, which suggests specific effects in the differentiation process. In line with this, apoptosis seemed to be stimulated at high doses of the AED. Regarding gene expression, valproic acid and topiramate promoted an increase in the expression of the functional genes COL1, ALP, and BMP2, whereas the remaining AED elicit an opposite response. The intracellular mechanisms modulated by the different AED were diverse. The MEK pathway seemed to be particularly important in the presence of valproic acid and gabapentin, and not involved in osteogenesis in the presence of carbamazepine and topiramate. Valproic acid, carbamazepine, and gabapentin elicited an upregulation of PKC, whereas all tested AED, except carbamazepine promoted a downregulation of the JNK pathway. As stated above, the analysis of specific changes in intracellular proteins involved in the tested pathways is underway.

A couple of previously published studies aimed to investigate the effects of valproic acid on bone cells, both with osteoblastic cell lines. It was observed that it stimulated cell proliferation and differentiation of mouse MC3T3-E1 osteoblastic cell line (Schroeder \& Westendorf, 2005). On the other hand, on human osteoblastic cell line hFOB 1.19, valproic acid seemed to cause a decrease in collagen type 1 and osteonectin production, but did not affect RANKL production (Humphrey et al., 2013). These observations suggest that valproic acid may be an important modulator of osteogenesis. In addition, valproic acid promoted a decrease in the proliferation of a mouse mesenchymal stem cell line (С3H10T1/2), at concentrations higher than $10^{-4} \mathrm{M}$ (Hatakeyama et al., 2011). At present, there are no reports aiming to characterize the effects of other AED, in addition to valproic acid, on osteoblastic cells, neither studies conducted with AED and osteoclastic cells.

In conclusion, it was demonstrated that the different AED can directly modulate human osteoclastogenesis and osteoblastogenesis, which may account, at least partially, to some of the potential deleterious effects of AED in bone metabolism. Among the tested $A E D$, valproic acid appeared to have the ability to interfere positively with both processes, which suggests a possible increase in bone turnover rate. The remaining AED displayed an inhibition of both processes (carbamazepine, gabapentin, and lamotrigine) or an inhibition of osteoclastogenesis and a stimulation of osteoblastogenesis (topiramate). This is the first report describing the direct effects of different AED on human primary bone cells, which is a very important issue, because these drugs are usually consumed in longterm therapeutics, with acknowledged in vivo effects in bone tissue. Understanding how AED affect bone tissue metabolism and integrity may help to develop new drugs and adjust therapeutics to each patient individual characteristics aiming to better clinical outcomes with minimal bone tissue-associated consequences.

\section{ACKNOWLEDGMENTS}

CLSM observation was performed at Advanced Light Microscopy, IBMC, University of Porto (IBMC.INEB) under the responsibility of Dr. Paula Sampaio. Partial financial support from the European Union (FEDER funds POCl/01/0145/FEDER/007265) and National Funds (FCT/MEC, Fundação para a Ciência e Tecnologia and Ministério da Educação e Ciência) under the Partnership Agreement PT2020 UID/ QUI/50006/2013. Thanks are also due to "Comissão de Coordenação e Desenvolvimento Regional do Norte (CCDRN)/NORTE2020/ Portugal 2020" for funding through project DESignBIOtechHealth (ref. Norte-01-0145-FEDER-000024).

\section{CONFLICT OF INTERESTS}

Authors declare that they have no conflict of interests. 


\section{AUTHOR CONTRIBUTIONS}

Sara Rocha conducted the experiments, Ricardo Ferraz designed and conducted the experiments, Cristina Prudêncio designed the experiments and wrote the paper, Maria Helena Fernandes designed the experiments and wrote the paper, and João Costa-Rodrigues designed and conducted the experiments and wrote the paper.

\section{REFERENCES}

Abou-Khalil, B. W. (2005). Making sense of lamotrigine serum levels. Epilepsy currents / American Epilepsy Society, 5, 115-115.

Albaghdadi, O., Alhalabi, M. S., Alourfi, Z., \& Youssef, L. A. (2016). Bone health and vitamin $D$ status in young epilepsy patients on valproate monotherapy. Clinical Neurology and Neurosurgery, 146, 52-56.

Ali, I. I., Herial, N. A., Orris, M., Horrigan, T., \& Tietjen, G. E. (2011). Migraine prophylaxis with topiramate and bone health in women. Headache, 51, 613-616.

Andress, D. L., Ozuna, J., Tirschwell, D., Grande, L., Johnson, M., Jacobson, A. F., \& Spain, W. (2002). Antiepileptic drug-induced bone loss in young male patients who have seizures. Archives of Neurology, 59, 781-786.

Bradford, M. M. (1976). A rapid and sensitive method for the quantitation of microgram quantities of protein utilizing the principle of proteindye binding. Analytical Biochemistry, 72, 248-254.

Carbone, L. D., Johnson, K. C., Robbins, J., Larson, J. C., Curb, J. D., Watson, K., \& Gass, M., et al. (2010). Antiepileptic drug use, falls, fractures, and BMD in postmenopausal women: Findings from the women's health initiative (WHI). Journal of Bone and Mineral Research, 25, 873-881.

Coppola, G., Fortunato, D., Auricchio, G., Mainolfi, C., Operto, F. F., \& Signoriello, G., et al. (2009). Bone mineral density in children, adolescents, and young adults with epilepsy. Epilepsia, 50, 2140-2146.

Costa-Rodrigues, J., Carmo, S., Perpetuo, I. P., Monteiro, F. J., \& Fernandes, M. H. (2016b). Osteoclastogenic differentiation of human precursor cells over micro- and nanostructured hydroxyapatite topography. Biochimica et Biophysica Acta/General Subjects, 1860, 825-835.

Costa-Rodrigues, J., Martins, E. G., \& Fernandes, M. H. (2012). Induced osteoclastogenesis by fluoroquinolones in unstimulated and stimulated human osteoclast precursor cells. Bone, 51, 17-27.

Costa-Rodrigues, J., Reis, S., Castro, A., \& Fernandes, M. H. (2016a). Bone anabolic effects of soluble $\mathrm{Si}$ : In vitro studies with human mesenchymal stem cells and CD14+ osteoclast precursors. Stem Cells International, 2016, 5653275-12.

Costa-Rodrigues, J., Silva, A., Santos, C., Almeida, M. M., Costa, M. E., \& Fernandes, M. H. (2014). Complex effect of hydroxyapatite nanoparticles on the differentiation and functional activity of human pre-osteoclastic cells. Journal of Biomedical Nanotechnology, 10, 3590-3600.

Ecevit, C., Aydogan, A., Kavakli, T., \& Altinoz, S. (2004). Effect of carbamazepine and valproate on bone mineral density. Pediatric Neurology, 31, 279-282.

Elliott, J. O., Jacobson, M. P., \& Haneef, Z. (2007). Homocysteine and bone loss in epilepsy. Seizure, 16, 22-34.

Fan, H. C., Lee, H. S., Chang, K. P., Lee, Y. Y., Lai, H. C., Hung, P. L., \& Lee, H. F., et al. (2016). The impact of anti-epileptic drugs on growth and bone metabolism. International Journal of Molecular Sciences, 17, E1242.

Gniatkowska-Nowakowska, A. (2010). Fractures in epilepsy children. Seizure, 19, 324-325.
Guo, C. Y., Ronen, G. M., \& Atkinson, S. A. (2001). Long-term valproate and lamotrigine treatment may be a marker for reduced growth and bone mass in children with epilepsy. Epilepsia, 42, 1141-1147.

Hamed, S. A., Moussa, E. M., Youssef, A. H., Abd ElHameed, M. A., \& NasrEldin, E. (2014). Bone status in patients with epilepsy: Relationship to markers of bone remodeling. Frontiers in Neurology, 5, 142.

Hant, F. N., \& Bolster, M. B. (2016). Drugs that may harm bone: Mitigating the risk. Cleveland Clinic Journal of Medicine, 83, 281-288.

Haroutiunian, S., Ratz, Y., Rabinovich, B., Adam, M., \& Hoffman, A. (2009). Valproic acid plasma concentration decreases in a dose-independent manner following administration of meropenem: A retrospective study. Journal of Clinical Pharmacology, 49, 1363-1369.

Hatakeyama, Y., Hatakeyama, J., Takahashi, A., Oka, K., Tsuruga, E., \& Inai, T., et al. (2011). The effect of valproic Acid on mesenchymal pluripotent cell proliferation and differentiation in extracellular matrices. Drug Target Insights, 5, 1-9.

Humphrey, E. L., Morris, G. E., \& Fuller, H. R. (2013). Valproate reduces collagen and osteonectin in cultured bone cells. Epilepsy Research, 106, 446-450.

Jette, N., Lix, L. M., Metge, C. J., Prior, H. J., McChesney, J., \& Leslie, W. D. (2011). Association of antiepileptic drugs with nontraumatic fractures: A population-based analysis. Archives of Neurology, 68, 107-112.

Kim, S. H., Lee, J. W., Choi, K. G., Chung, H. W., \& Lee, H. W. (2007). A 6-month longitudinal study of bone mineral density with antiepileptic drug monotherapy. Epilepsy \& Behavior, 10, 291-295.

Lazzari, A. A., Dussault, P. M., Thakore-James, M., Gagnon, D., Baker, E., \& Davis, S. A., et al. (2013). Prevention of bone loss and vertebral fractures in patients with chronic epilepsy--antiepileptic drug and osteoporosis prevention trial. Epilepsia, 54, 1997-2004.

Lee, H. S., Wang, S. Y., Salter, D. M., Wang, C. C., Chen, S. J., \& Fan, H. C. (2013). The impact of the use of antiepileptic drugs on the growth of children. BMC Pediatrics, 13, 211.

Lee, R. H., Lyles, K. W., \& Colon-Emeric, C. (2010). A review of the effect of anticonvulsant medications on bone mineral density and fracture risk. The American Journal of Geriatric Pharmacotherapy, 8, 34-46.

Lin, C. M., Fan, H. C., Chao, T. Y., Chu, D. M., Lai, C. C., \& Wang, C. C., et al. (2016). Potential effects of valproate and oxcarbazepine on growth velocity and bone metabolism in epileptic children- a medical center experience. BMC Pediatrics, 16, 61.

Lyseng-Williamson, K. A., \& Yang, L. P. (2007). Topiramate: A review of its use in the treatment of epilepsy. Drugs, 67, 2231-2256.

Mantegazza, M., Curia, G., Biagini, G., Ragsdale, D. S., \& Avoli, M. (2010). Voltage-gated sodium channels as therapeutic targets in epilepsy and other neurological disorders. Lancet Neurology, 9, 413-424.

O'Dougherty, M., Wright, F. S., Cox, S., \& Walson, P. (1987). Carbamazepine plasma concentration. Relationship to cognitive impairment. Archives of Neurology, 44, 863-867.

Oner, N., Kaya, M., Karasalihoglu, S., Karaca, H., Celtik, C., \& Tutunculer, F. (2004). Bone mineral metabolism changes in epileptic children receiving valproic acid. Journal of Paediatrics and Child Health, 40, 470-473.

Pitetzis, D. A., Spilioti, M. G., Yovos, J. G., \& Yavropoulou, M. P. (2017). The effect of VPA on bone: From clinical studies to cell cultures-The molecular mechanisms revisited. Seizure, 48, 36-43.

Popov, T. V., Maricic, L. C., Prosen, H., \& Voncina, D. B. (2013). Determination of topiramate in human plasma using liquid chromatography tandem mass spectrometry. Acta Chimica Slovenica, 60, 144-150.

Rattya, J., Vainionpaa, L., Knip, M., Lanning, P., \& Isojarvi, J. I. (1999). The effects of valproate, carbamazepine, and oxcarbazepine on growth and sexual maturation in girls with epilepsy. Pediatrics, 103, 588-593.

Rauchenzauner, M., Griesmacher, A., Tatarczyk, T., Haberlandt, E., Strasak, A., Zimmerhackl, L. B., \& Falkensammer, G., et al. (2010). Chronic antiepileptic monotherapy, bone metabolism, and body composition in non-institutionalized children. Developmental Medicine and Child Neurology, 52, 283-288. 
Rose, M. A., \& Kam, P. C. (2002). Gabapentin: Pharmacology and its use in pain management. Anaesthesia, 57, 451-462.

Sato, Y., Kondo, I., Ishida, S., Motooka, H., Takayama, K., Tomita, Y., \& Maeda, $H$., et al. (2001). Decreased bone mass and increased bone turnover with valproate therapy in adults with epilepsy. Neurology, 57, 445-449.

Schroeder, T. M., Nair, A. K., Staggs, R., Lamblin, A. F., \& Westendorf, J. J. (2007). Gene profile analysis of osteoblast genes differentially regulated by histone deacetylase inhibitors. BMC Genomics, 8, 362.

Schroeder, T. M., \& Westendorf, J. J. (2005). Histone deacetylase inhibitors promote osteoblast maturation. Journal of Bone and Mineral Research, 20, 2254-2263.

Serin, H. M., Koc, Z. P., Temelli, B., \& Esen, I. (2015). The bone mineral content alterations in pediatric patients medicated with levetiracetam, valproic acid, and carbamazepine. Epilepsy \& Behavior, 51, 221-224.

Shen, C., Chen, F., Zhang, Y., Guo, Y., \& Ding, M. (2014). Association between use of antiepileptic drugs and fracture risk: A systematic review and meta-analysis. Bone, 64, 246-253.

Siddiqui, J. A., \& Partridge, N. C. (2016). Physiological bone remodeling: Systemic regulation and growth factor involvement. Physiology (Bethesda, Md.), 31, 233-245.

Song, X. Q., Wang, Z. P., Bao, K. R., Zhang, J. M., Wu, J., \& Yan, C. H., et al. (2005). Effect of carbamazepine and valproate on bone metabolism in children with epilepsy. Zhonghua er ke za zhi. Chinese Journal of Pediatrics, 43, 728-732.

Soysa, N. S., \& Alles, N. (2016). Osteoclast function and bone-resorbing activity: An overview. Biochemical and Biophysical Research Communications, 476, 115-120.

Triantafyllou, N., Lambrinoudaki, I., Armeni, E., Evangelopoulos, E. M., Boufidou, F., \& Antoniou, A., et al. (2010). Effect of long-term valproate monotherapy on bone mineral density in adults with epilepsy. Journal of the Neurological Sciences, 290, 131-134.

Tsukahara, H., Kimura, K., Todoroki, Y., Ohshima, Y., Hiraoka, M., \& Shigematsu, Y., et al. (2002). Bone mineral status in ambulatory pediatric patients on long-term anti-epileptic drug therapy. Pediatria Internazionale, 44, 247-253.

Valenti, M. T., Dalle Carbonare, L., \& Mottes, M. (2016). Osteogenic differentiation in healthy and pathological conditions. International Journal of Molecular Sciences, 18, 41.

Verrotti, A., Agostinelli, S., Coppola, G., Parisi, P., \& Chiarelli, F. (2010). A 12-month longitudinal study of calcium metabolism and bone turnover during valproate monotherapy. European Journal of Neurology, 17, 232-237.

Verrotti, A., Greco, R., Latini, G., Morgese, G., \& Chiarelli, F. (2002). Increased bone turnover in prepubertal, pubertal, and postpubertal patients receiving carbamazepine. Epilepsia, 43, 1488-1492.

Zhang, Y., Zheng, Y. X., Zhu, J. M., Zhang, J. M., \& Zheng, Z. (2015). Effects of antiepileptic drugs on bone mineral density and bone metabolism in children: A meta-analysis. Journal of Zhejiang University. Science. B, 16, 611-621. 